\title{
Estudo clinicopatológico de 106 adenomas pleomórficos de glândula salivar maior
}

\author{
A clinicopathologic study of 106 pleomorphic adenomas of the major salivary glands
}

Jerlucia Cavalcanti das Neves'; Maria do Carmo Abreu e Lima²; Ana Paula Veras Sobral ${ }^{3}$

\begin{abstract}
unitermos
resumo

Adenoma pleomórfico

Introdução: O adenoma pleomórfico (AP) é o mais comum dos tumores das glândulas salivares. Transformação maligna pode ocorrer após recorrências ou em casos com longo tempo de evolução.

Patologia Objetivo: Analisar os aspectos clinicoepidemiológicos e anatomopatológicos do AP de glândula salivar

Glândulas salivares maior, principalmente os considerados indício de transformação maligna. Material e método: Foram avaliados 106 casos de AP pela pesquisa retrospectiva nos prontuários clínicos e revisão das preparações maiores histológicas. Resultados: A média de idade dos pacientes foi de 39,51 anos, houve predomínio do Transformação maligna sexo feminino $(69,5 \%)$, a glândula parótida foi a mais acometida $(86,8 \%)$ e o tempo de evolução foi superior a um ano em $76,74 \%$ dos casos, com tamanho tumoral de $3,48 \mathrm{~cm}$ em média. A avaliação histopatológica demonstrou cápsula tumoral incompleta e delgada em $49 \%$ dos casos. Protrusões para a cápsula ou extensão extracapsular foram infreqüentes, $11,32 \%$ e $8,49 \%$, respectivamente. A matriz extracelular variou entre mixocondróide (41,5\%), mixóide (36,8\%), condróide $(3,8 \%)$ e fibrótica $(1,9 \%)$, tendo sido observadas associações entre os diversos tipos. O componente epitelial/mioepitelial constituiu $50 \%$ ou mais do tumor em $65,1 \%$ dos casos e estava disposto em arranjos cordonal (86,8\%), ductal $(81,1 \%)$, sólido (40,6\%), cístico (20,7\%) e em "paliçada" (8,49\%). Metaplasia escamosa ocorreu em $16,04 \%$ dos casos. Alterações histopatológicas relacionadas com transformação maligna foram incomuns: hialinização extensa $(4,7 \%)$ e necrose $(0,9 \%)$. Conclusões: confirma-se a diversidade histopatológica do AP. Características associadas a transformação maligna não foram freqüentes na amostra.
\end{abstract}

\footnotetext{
Introduction: Pleomorphic adenomas (PA) are the most common histologic type of salivary gland tumors. Malignant transformation may occur in the setting of recurrent or longstanding disease. Objective: Evaluate the clinicopathologic characteristics of PA of the major salivary glands, particularly tumors showing signs of malignant transformation. Material and methods: The clinical and histopathologic characteristics of 106 cases of PA were evaluated retrospectively. Results: The mean patient age was 39.5 years. There was a predominance of female patients (69.5\%). The parotid was the most commonly affected gland (86.8\%). The tumor had been present for over a year in $76.74 \%$ of the cases, with a mean size of $3.48 \mathrm{~cm}$. Histologic examination revealed a thin and incomplete capsule in $49 \%$ of the cases; however, protrusions into the capsule or extracapsular extension were infrequent $(11.32 \%$ and $8.49 \%$, respectively). The extracellular matrix varied from myxochondroid (41.5\%), myxoid (36.8\%), chondroid (3.8\%) and fibrotic (1.9\%); coexisting patterns were observed. The myoepithelial-epithelial component corresponded to $50 \%$ or more of the tumor in $65.1 \%$ of the cases, and was solid (40.6\%), cystic (20.7\%), arranged in cords (86.8\%), ducts (81.1\%) and had a palisading configuration (8.49\%). Squamous metaplasia was present in $16.04 \%$ of the cases. Features associated with malignant transformation were incommon: extensive hyalinization $(4,7 \%)$ and necrosis (0,9\%). Conclusion: The histopathologic diversity of PA was confirmed. Features associated to malignant transformation were unfrequent.
}

\footnotetext{
1. Cirurgiã-dentista; especialista em Estomatologia pela Universidade Federal de Pernambuco (UFPE); aluna do Programa de Pós-Graduação em Patologia da UFPE; professora de Patologia Oral Semiologia da Faculdade de Odontologia do Recife (FOR).

2. Professora associada do Departamento de Anatomia Patológica da UFPE e do Programa de Pós-Craduação em Patologia da UFPE; doutora em Medicina pela UFPE.

3. Professora-adjunta da disciplina de Patologia Bucal da Faculdade de Odontologia de Pernambuco da Universidade de Pernambuco (FOP/UPE); doutora em Patologia Bucal pela Faculdade de Odontologia da Universidade de São Paulo (FOUSP).

Trabalho realizado no Departamento de Patologia do Hospital de Câncer de Pernambuco (HCP) e no Laboratório de Patologia Bucal da FOP/UPE.
} 


\section{Introdução}

O adenoma pleomórfico é o mais comum entre os tumores das glândulas salivares maiores e menores. A parótida é a sede mais freqüente, sendo responsável por $85 \%$ a $90 \%$ dos casos, embora possa ocorrer em qualquer das glândulas salivares. Possui características clínicas e morfológicas bem conhecidas. Entretanto sua patogênese ainda é incerta, assim como a expressão de oncogenes e fatores que influenciam sua transformação maligna ${ }^{(1,4,5,10,}$ $14,16,18,24,20)$. A transformação maligna ocorre principalmente após recorrências do tumor ou em casos nos quais o tumor apresente um longo tempo de evolução. $O$ espectro de características histológicas do componente maligno é amplamente variável( ${ }^{(3,14)}$.

Embora critérios para a distinção entre elementos benignos e malignos em carcinomas ex-adenoma pleomórfico tenham sido adequadamente descritos, existem poucas tentativas de se identificarem características clínicas e histológicas em adenomas pleomórficos indicando o risco de transformação maligna $a^{(2)}$.

Esse estudo tem por objetivo analisar os aspectos clinicoepidemiológicos e anatomopatológicos em adenomas pleomórficos de glândula salivar maior, principalmente aqueles considerados de risco para a transformação maligna, correlacionando-os.

\section{Material e método}

O material para a presente pesquisa provém dos arquivos de blocos e lâminas do Departamento de Patologia do Hospital de Câncer de Pernambuco (HCP) e consiste em 106 casos de adenoma pleomórfico diagnosticados no período de janeiro de 2001 a dezembro de 2004.

Dados de interesse demográfico, clínicos, tratamento, recorrência e óbito foram coletados mediante pesquisa retrospectiva, utilizando-se arquivos dos laudos anatomopatológicos do Departamento de Patologia e prontuários clínicos dos pacientes oriundos do Departamento de Cabeça e Pescoço do HCP.

As preparações histológicas coradas em hematoxilina e eosina(HE) referentes aos casos selecionados foram revisadas em conjunto por dois avaliadores para confirmação do diagnóstico e determinação da freqüência dos achados histopatológicos. Os critérios morfológicos para o diagnóstico de adenoma pleomórfico adotados foram os da Organização Mundial da Saúde (OMS) $)^{(11)}$. Recortes a partir dos blocos originais foram obtidos quando necessário.
$\mathrm{Na}$ análise anatomopatológica foram observadas as seguintes características microscópicas: cápsula tumoral, contornos do tumor, margens cirúrgicas, proporção dos componentes (matriz extracelular e elementos epiteliais/ mioepiteliais), tipo de matriz extracelular, morfologia celular, metaplasia, arranjo das células tumorais, presença de alterações associadas a transformação maligna (necrose focal, hialinização extensa e mitoses atípicas) e outras alterações histológicas (calcificações, cristalóides, infiltração adiposa, enfarte e fibrose).

Quanto à matriz extracelular, quantificamos a sua proporção em relação ao componente epitelial/mioepitelial no tumor, classificando-a em escassa, moderada e abundante. A quantidade de matriz extracelular de até $45 \%$ de toda a estrutura tumoral foi considerada escassa; de $45 \%$ a $70 \%$, moderada; e acima de 70\%, abundante.

$\mathrm{Na}$ análise estatística, os dados quantitativos foram resumidos por meio dos valores mínimo, máximo, mediana, média e desvio padrão. Os dados qualitativos foram resumidos através de freqüências absoluta e relativa percentual. Empregou-se o teste exato de Fisher para avaliar comparativamente dois ou mais grupos quanto à homogeneidade de proporções. Optou-se por esse teste porque a maioria das tabelas de contingência apresentava valores esperados menores que cinco em várias células. Para comparar dados quantitativos em dois grupos foi utilizado o teste $t$ de Student, e para comparar três ou mais grupos, a análise da variância (ANOVA). Em todos os testes foi adotado o nível de significância de 0,05.

\section{Resultados}

\section{Achados epidemiológicos e clínicos}

A idade dos pacientes em nossa amostra variou de 15 a 84 anos, com média de 39,5 anos. O sexo feminino foi o mais acometido, representando $69,5 \%$ (73) dos casos, enquanto o masculino representou $30,5 \%$ (32).

A queixa principal dos pacientes foi relatada em 45 dos 106 casos estudados. O incômodo relacionado ao tamanho do tumor representou $84,5 \%$ (38) das queixas, enquanto $13,3 \%$ (6) estavam relacionadas a sintoma de dor.

Os hábitos de tabagismo e/ou etilismo foram relatados por $33(31,1 \%)$ dos 106 pacientes. O tabagismo foi predominante, constituindo $75,8 \%$ (25), tendo sido o etilismo relatado por apenas dois (6\%) pacientes. A associação entre os dois hábitos foi observada em 18,9\% (6). 
A glândula salivar mais acometida foi a parótida (92; $86,8 \%)$, seguida pela submandibular $(13 ; 12,3 \%)$ e pelas glândulas sublinguais $(1 ; 0,9 \%)$.

O tempo de evolução do tumor foi referido em 86 dos 106 casos estudados; destes, 66 (76,7\%) apresentaram tempo de evolução superior a um ano e 12 (13,9\%), entre seis meses e um ano.

A excisão cirúrgica foi o tratamento realizado em todos os casos estudados, sendo $83 \%$ (88) de cirurgia com remoção total da glândula salivar e 17\% (18) de cirurgia parcial, com remoção apenas da porção glandular acometida pelo tumor. Nenhum paciente foi a óbito em decorrência do tumor ou do tratamento.

A neoplasia foi considerada primária em 96,2\% (102) dos casos, e em 3,8\% (4) foi considerada recidiva. Os tumores recidivantes foram assim julgados de acordo com relatos clínicos de cirurgias e exames anatomopatológicos prévios cujo diagnóstico foi de adenoma pleomórfico. Os quatro casos de recidiva ocorreram na glândula parótida em intervalo de tempo superior a dois anos da exérese do tumor primário.

\section{Achados microscópicos}

A cápsula tumoral apresentou-se incompleta (Figura 1A) e delgada em 52 casos (49,1\%), sendo completa e delgada em 33 (31,1\%) (Figura 1B). Em 11,3\% (12) dos casos foram observadas lingüetas de tecido tumoral projetandose para a cápsula (Figura 1C), e em 8,5\% (9) ocorreu extensão extracapsular do tumor. O contorno do tumor foi nodular em 50\% (53) dos casos e lobulado em 26 (24,5\%). As recidivas (4;3,8\%) (Figura 1D) se caracterizaram por arranjo em nódulos múltiplos dispersos em meio a tecido fibroadiposo.

A quantidade de matriz extracelular foi escassa em 32 (30,2\%) casos, moderada em 37 (34,9\%) e abundante em 37 (34,9\%). Houve tumores hipercelulares, constituindo $30,2 \%$ (32), caracterizados por componente epitelial/mioepitelial dominante. $O$ tipo de matriz extracelular prevalente nos casos estudados foi o mixocondróide, representando 41,5\% (44); o tipo mixóide foi observado em $36,8 \%$ dos casos (39); outros tipos de matriz, embora pouco freqüentes, foram encontrados, como os tipos condróide puro (quatro; 3,8\%) e fibrótico (dois; $1,9 \%$ ).

O arranjo histológico das células epiteliais/mioepiteliais foi bastante variado nessa amostra. Houve predomínio dos arranjos cordonal (92; 86,8\%) (Figura 2A) e ductal
(Figura 2B) (86; 81,1\%). Padrões sólidos foram observados em 43 (40,6\%) tumores, havendo também a formação de cistos em alguns casos (22; 20,7\%). O arranjo celular em paliçada (Figura 2C) foi incomum, observado em nove $(8,5 \%)$ casos. Metaplasia escamosa (Figura 2D) foi observada em $16 \%$ (17) dos casos.

Em relação às alterações histológicas relacionadas com a transformação maligna, descritas na Tabela 1, foi observado que $57(48,1 \%)$ casos apresentaram hialinização, sendo considerada discreta em $49(46,2 \%)$, moderada em três $(2,8 \%)$ e extensa em cinco $(4,7 \%)$ (Figura 3A); um caso $(0,9 \%)$ apresentou necrose e $48(45,3 \%)$ dos tumores não demonstraram nenhuma das alterações consideradas indícios de transformação maligna.

A fibrose (Figura 3B) foi um achado histopatológico comum entre os tumores estudados, tendo sido encontrada em $70,7 \%$ (75) deles. Outros achados, como calcificações (Figura 3C) (sete; 6,6\%), cristais de colesterol (dois; 1,9\%)

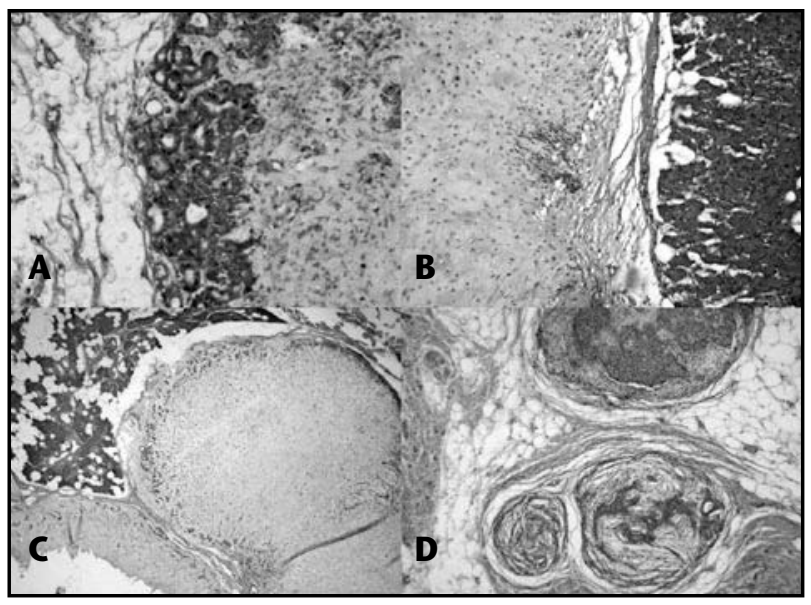

Figura 1 - A: Cápsula incompleta (HE, 100x); B: cápsula delgada (HE, 100x); C: Protrusões para a cápsula (HE, 25x); D: Padrão de recidiva (HE, 100x)

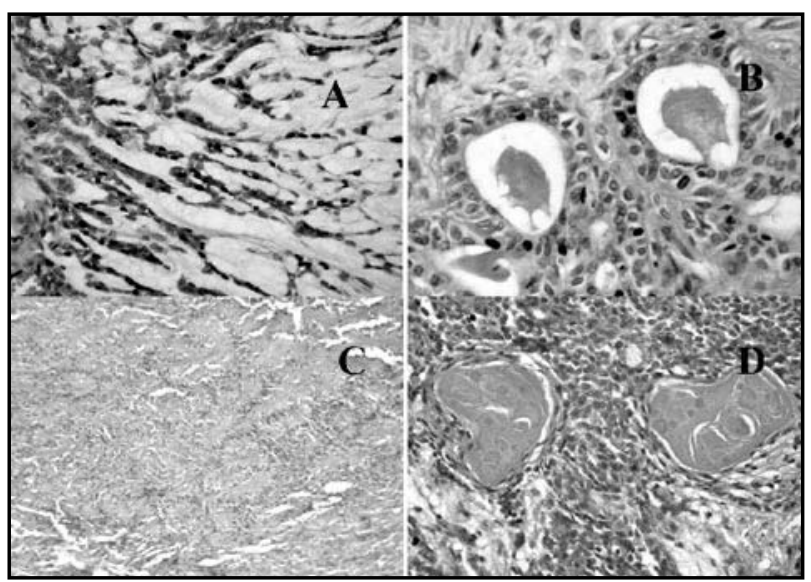

Figura 2 - A: Arranjo cordonal (HE, 400x); B: arranjo ductal (HE, 400x); C: paliçada (HE, 100x); D: metaplasia escamosa (HE, 400x) 


\section{Tabela 1 Freqüência das alterações histopatológicas relacionadas à transformação maligna}

\begin{tabular}{lcc}
\hline Alterações relacionadas à transformação maligna & Freqüência $(n)$ & Porcentagem $(\%)$ \\
Hialinização extensa & 5 & 4,7 \\
Hialinização moderada & 3 & 2,8 \\
Hialinização discreta & 49 & 46,2 \\
Necrose & 1 & 0,9 \\
Sem alteração & 48 & 45,3 \\
Total & 106 & 100 \\
\hline
\end{tabular}

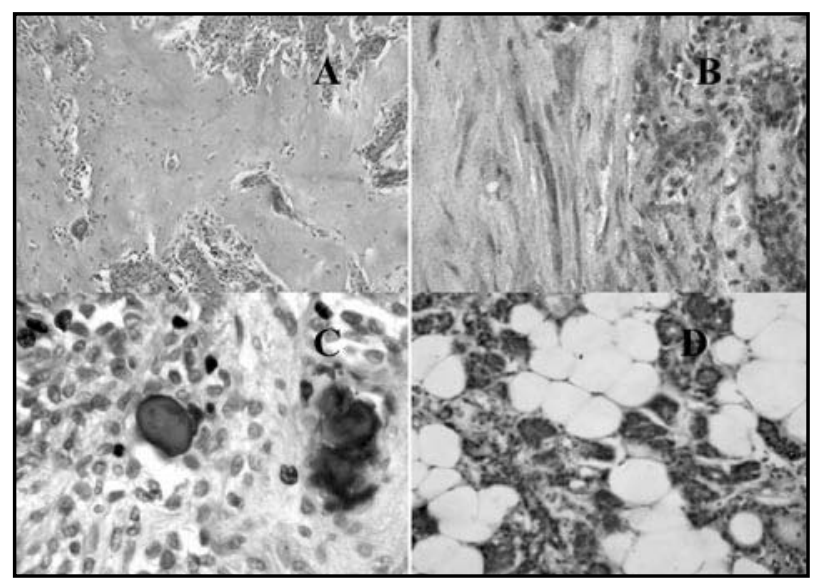

Figura 3 - A: Hialinização $(H E, 100 x)$; B: fibrose (HE, 400x); C: calcificação (HE, 400x); D: infiltração adiposa (HE, 400x)

e infiltração adiposa (Figura 3D) (dois; 1,9\%) também foram observados.

O tecido glandular adjacente ao tumor estava presente em $95(89,6 \%)$ dos 106 casos estudados, encontrando-se sem alterações significativas em quatro (3,8\%). Em 78,3\% (83) dos casos foi observada infiltração adiposa, e em 2,8\% (três), inflamação crônica. Inflamação e infiltração adiposa foram observadas simultaneamente em 4,7\% (cinco) dos casos. A diferença entre as médias de idade e as alterações encontradas no tecido glandular adjacente ao tumor não foi estatisticamente significativa, como visto na Tabela 2.

Não houve associação estatisticamente significativa entre as características de idade e sexo em relação ao tipo e à quantidade de matriz extracelular, ou à presença de metaplasia na amostra, conforme demonstrado nas

Tabelas 3 e 4.

\section{Discussão}

O adenoma pleomórfico nas séries estudadas representa de $40 \%$ a $80 \%$ de todos os tumores salivares benignos ou malignos, tendo como sítio anatômico mais freqüente a glândula parótida $(5,10,12,15,16,19,20,21,27-29,31,34)$. Na amostra estudada observamos que a glândula parótida foi acometida em $86,8 \%$ (92), ocorrendo em menor freqüência nas glândulas submandibular (13; 12,3\%) e sublingual (um; 0,9\%). Esses resultados são concordantes com os já encontrados por nós na literatura.

Trata-se de um tumor comum na faixa etária compreendida entre os 40 e 60 anos de idade e que apresenta

\section{Tabela 2 dula salivar adjacente}

\begin{tabular}{lcccccc}
\hline \multirow{2}{*}{ Alteração tecidual da glândula salivar adjacente } & \multicolumn{7}{c}{ Estatísticas descritivas da idade } \\
Sem alteração & $n$ & Média & DP & Mínimo & Mediana & Máximo \\
Inflamação & 4 & 36 & 10,9 & 23 & 37,5 & 46 \\
Infiltração adiposa & 3 & 31 & 7,5 & 23 & 32 & 38 \\
Não observada & 82 & 39,9 & 14,4 & 15 & 38 & 84 \\
Inflamação e infiltração adiposa & 11 & 42,4 & 17,6 & 16 & 37 & 72 \\
Total & 5 & 35 & 15,2 & 20 & 30 & 58 \\
\hline
\end{tabular}

Teste $t$ de Student: $p=0,696$.

$D P$ : desvio padrão. 


\section{Tabela 3 Associação entre sexo e variáveis histopatológicas}

\begin{tabular}{|c|c|c|c|c|}
\hline \multirow{2}{*}{ Variáveis } & \multicolumn{2}{|c|}{ Sexo } & \multirow{2}{*}{ Total } & \multirow[b]{2}{*}{$p$} \\
\hline & Feminino & Masculino & & \\
\hline Tipo de matriz extracelular & & & & 0,903 \\
\hline Mixóide & $32(43,8 \%)$ & $15(46,9 \%)$ & $47(44,8 \%)$ & \\
\hline Mixocondróide & $37(50,7 \%)$ & $15(46,9 \%)$ & $52(49,5 \%)$ & \\
\hline Condróide & $3(4,1 \%)$ & $1(3,1 \%)$ & $4(3,8 \%)$ & \\
\hline Fibrótico & $1(1,4 \%)$ & $1(3,1 \%)$ & $2(1,9 \%)$ & \\
\hline Subtotal & $73(100 \%)$ & $32(100 \%)$ & $105(100 \%)$ & \\
\hline Quantidade de matriz extracelular & & & & 0,372 \\
\hline Escassa (até 45\%) & $25(34,2 \%)$ & $7(21,9 \%)$ & $32(30,5 \%)$ & \\
\hline Moderada (45\% a $70 \%$ ) & $23(31,6 \%)$ & $14(43,8 \%)$ & $37(35,2 \%)$ & \\
\hline Abundante (acima de $70 \%$ ) & $25(34,2 \%)$ & $11(34,4 \%)$ & $36(34,3 \%)$ & \\
\hline Subtotal & $73(100 \%)$ & $32(100 \%)$ & $105(100 \%)$ & \\
\hline Metaplasia & & & & 1 \\
\hline Sim & $11(15,1 \%)$ & $4(12,5 \%)$ & $15(14,3 \%)$ & \\
\hline Não & $62(84,9 \%)$ & $28(87,5 \%)$ & $90(85,7 \%)$ & \\
\hline Subtotal & $73(100 \%)$ & $32(100 \%)$ & $105(100 \%)$ & \\
\hline
\end{tabular}

Teste exato de Fisher.

Tabela 4

Principais estatísticas descritivas e comparação das médias da idade dos pacientes, de acordo com algumas variáveis histopatológicas

\begin{tabular}{lccccccc}
\hline \multirow{2}{*}{$\begin{array}{l}\text { Variáveis } \\
\text { Tipo de matriz extracelular }\end{array}$} & $n$ & Média & DP & Mínimo & Mediana & Máximo & $p$ \\
Mixóide & 47 & 41 & 15,2 & 18 & 38 & 84 & $0,357^{\dagger}$ \\
Mixocondróide & 52 & 38,8 & 13,8 & 15 & 38 & 76 & \\
Condróide & 4 & 28,5 & 12,3 & 16 & 27 & 44 & \\
Fibrótico & 2 & 45,5 & 12 & 37 & 45,5 & 54 & \\
Matriz extracelular & & & & & & & \\
Escassa (até 45\%) & 32 & 41,2 & 12 & 15 & 40 & 72 & $0,325^{\dagger}$ \\
Moderada (45\% a 70\%) & 37 & 40,9 & 16,5 & 15 & 38 & 76 & \\
Abundante (acima de 70\%) & 36 & 36,6 & 14 & 17 & 34,5 & 84 & \\
Metaplasia & & & & & & & \\
Sim & 15 & 34,3 & 15,4 & 15 & 34 & 76 & \\
Não & 90 & 40,4 & 14,2 & 16 & 38 & 84 & \\
\hline
\end{tabular}

${ }^{\dagger}$ Análise de variância (ANOVA); ‘†teste t de Student. DP: desvio padrão.

maior freqüência no sexo feminino $(5,10,15,16,19,27,29)$. Em nossa casuística foi observada uma média de idade semeIhante aos estudos epidemiológicos do tumor (39,5 anos), e o sexo feminino representou $69,5 \%(n=73)$ dos casos.
Corroboramos Forty e Wake ${ }^{(15)}$ e Garcia-Perla et al. ${ }^{(17)}$, que consideram esse tumor de ocorrência rara em crianças, já que a idade mínima encontrada nos 106 casos estudados foi de 15 anos. 
O tempo de evolução das lesões foi superior a um ano em $76,7 \%$ dos casos, caracterizando, portanto, crescimento lento ${ }^{(6,9,11,25)}$. Embora o adenoma pleomórfico seja considerado um tumor indolor ${ }^{(6,9,11,25)}$, foi observado em nosso trabalho que a sintomatologia dolorosa, apesar de pouco freqüente, representou a segunda queixa principal dos portadores dessa neoplasia, constituindo $13,3 \%$ (seis) das queixas relatadas. A principal queixa estava relacionada com o aumento de volume do tumor, observado em $38(84,4 \%)$ pacientes. Entretanto, dados sobre a anamnese e o exame clínico dos pacientes foram escassos nos prontuários, dificultando uma análise mais apurada das características clínicas do tumor.

Apesar de não existir associação comprovada entre hábitos como tabagismo e/ou etilismo e o surgimento do adenoma pleomórfico ${ }^{(6)}$, verificamos o tabagismo em 25 pacientes, representando $75,8 \%$, e o etilismo em apenas dois (6\%) pacientes, sendo a associação entre os dois hábitos observada em seis $(18,2 \%)$ casos, o que pode sugerir que o tabagismo esteja relacionado com o surgimento desses tumores. Entretanto, os dados obtidos não refletem a totalidade da amostra estudada, visto que apenas 33 dos 106 pacientes apresentaram esses hábitos.

O adenoma pleomórfico das glândulas salivares maiores é um tumor encapsulado, podendo esta cápsula ser incompleta, permitindo que, com o crescimento tumoral, ocorram protrusões do tumor para o interior da glândula salivar adjacente, fato considerado fator predisponente para a recorrência dessa neoplasia ${ }^{(6,10,15,23)}$. Em nosso estudo, a cápsula tumoral apresentou-se delgada em 80,2\% (85) dos casos, sendo incompleta na maioria das vezes $(52 ; 49,1 \%)$. Em apenas 11,3\% (12) dos casos foram observadas protrusões ou lingüetas de tecido tumoral permeando a cápsula, e em 8,5\% (nove) ocorreu extensão extracapsular do tumor. Entretanto, não foi observada recidiva dos tumores considerados primários em nosso estudo, com acompanhamento mínimo de 38 meses e máximo de cinco anos. Esse fato pode ser atribuído ao tratamento cirúrgico com remoção total da glândula salivar acometida, aplicado à maioria dos tumores no HCP e preconizado na literatura ${ }^{(8,16,33)}$.

Os adenomas pleomórficos são conhecidos por sua

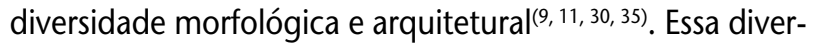
sidade ficou evidente em nosso estudo, no qual foi possível encontrar tumores extremamente celulares (32; 30,2\%) em contraste com outros em que a matriz extracelular predominou $(37 ; 34,9 \%)$.

O componente epitelial/mioepitelial pode estar arranjado em padrões diversos: com formação de ductos, ácinos pequenos, ou túbulos, cordões irregulares, faixas ou camadas de células ${ }^{(6,10)}$. Acredita-se que as células mioepiteliais compreendem o principal tipo celular encontrado no parênquima dos adenomas pleomórficos, sendo implicadas como responsáveis pelas variações histológicas do estroma neoplásico ${ }^{(7,32)}$. Tais células podem arranjar-se de forma reticular fina ou em cordões de células fusiformes, ou até mesmo em paliçada, adquirindo aparência semelhante ao schwannoma ${ }^{(11)}$. Arranjos celulares diversos puderam ser observados em nosso estudo, com predomínio do cordonal (92; $86,8 \%)$ e do ductal $(86 ; 81,1 \%)$ na maioria dos tumores. Arranjos sólidos também foram observados, porém com menor freqüência. A disposição celular em paliçada foi incomum, observada em apenas nove $(8,5 \%)$ casos. Em muitos casos notou-se uma tendência de os elementos epiteliais/mioepiteliais se disporem em maior concentração na periferia do nódulo, logo abaixo da cápsula.

Estruturas ductais presentes nos casos estudados foram mais numerosas nas áreas ricamente epiteliais, e em alguns casos tais estruturas apresentaram-se dilatadas e com lúmen bem definido, freqüentemente contendo material eosinofílico colóide-símile em seu interior, tal como descrito na literatura(11).

Metaplasia escamosa, algumas vezes com formação de "pérolas" de queratina, pode ser vista nos ductos e lençóis de células e, ocasionalmente, há metaplasia mucosa ou presença de células claras ${ }^{(11)}$. A metaplasia escamosa foi observada em 16\% (17) dos casos da nossa amostra. Embora observássemos ocasional deposição de material mucóide, não ocorreu metaplasia mucosa. Houve presença de células claras em 38 (35,8\%) casos.

A maioria dos casos estudados $(69 ; 65,1 \%)$ apresentou componente epitelial/mioepitelial, constituindo $50 \%$ ou mais do tumor, e 30,2\% (32) dos casos apresentaram o componente epitelial/mioepitelial constituindo $80 \%$ ou mais do tumor. Todavia, outros autores consideram que apenas $12 \%$ a $15 \%$ dessas neoplasias têm um componente epitelial/mioepitelial que constitui mais de $80 \%$ da totalidade do tumor ${ }^{(9,26)}$. A celularidade de adenomas pleomórficos, entretanto, não é considerada de significância para o prognóstico tumoral(11).

A presença de necrose focal, mitoses atípicas e extensa hialinização(6,10,22) sugere a possibilidade de transformação maligna em adenoma pleomórfico. Dessas alterações, a hialinização extensa foi encontrada em cinco $(4,7 \%)$ casos, necrose focal em apenas um $(0,9 \%)$ caso e mitoses atípicas não foram observadas.

Em nossa amostra, apenas 5,6\% (seis) dos casos apresentaram características histopatológicas descritas na literatura 
como indícios de transformação maligna, sugerindo que essas características não são freqüentes nesses tumores.

\section{Conclusões}

Diante do exposto podemos concluir que o adenoma pleomórfico de glândula salivar maior é um tumor de grande variedade histopatológica, tendo como característica a ocorrência de diversos arranjos arquiteturais, estruturais e variações de celularidade por vezes em um mesmo tumor.

As suas características clínicas e demográficas já estão bem estabelecidas na literatura, porém é necessário que estudos mais detalhados dos padrões histopatológicos sugestivos de transformação maligna sejam realizados.

As alterações descritas na literatura como indícios de transformação maligna foram pouco comuns em nossa amostra, sugerindo que essas características não são freqüentes nesses tumores.

\section{Agradecimentos}

Ao Dr. Adonis de Carvalho, chefe do Departamento de Patologia do HCP, e aos funcionários do Laboratório de Patologia do HCP, pela oportunidade de trabalharmos com o material cedido dos arquivos deste serviço.

\section{Referências}

1. ALVES, F. A. et al. PCNA, Ki-67 and P53 expressions in submandibular salivary gland tumors. Int J Oral Maxillofac Surg, v. 33, n. 6, p. 593-97, 2004.

2. AUCLAIR, P. L.; ELLIS, G. L. Atypical features in salivary gland mixed tumors: their relationship to malignant transformation. Mod Pathol, v. 9, p. 652-7, 1996.

3. BATSAKIS, J. G. Carcinoma ex-pleomorphic adenoma's "Big Tent" has room for low and high grade tumors. Adv Anat Pathol, v. 4, p. 176-8, 1997.

4. BERROCAL JR, G. et al. Mixed tumor (pleomorphic adenoma) of head and neck. Types and atypical patterns. An Otorrinolaringol Ibero Am, v. 27, n. 4, p. 333-40, 2000.

5. BURZLAFF, J. B.; PURICELLI, E. Estudo imuno-histoquímico em adenomas pleomórficos das glândulas salivares. Rev Fac Odontol Porto Alegre, v. 37, n. 2, p. 19-24, 1996.

6. COTRAN, R. S.; KUMAR, V.; COLLINS, T. Robbins: patologia estrutural e funcional. 6. ed. Rio de Janeiro: Guanabara Koogan, 2000.

7. DARDICK, I. Color atlas-text of salivary gland tumor pathology. New York: Igakushoin, 1996.

8. DOUGLAS, J. G. et al. Neutron radiotherapy for recurrent pleomorphic adenomas of major salivary glands. Head Neck, v. 23, n. 12, p. 1037-42, 2001.

9. ELLIS, G. L.; AUCLAIR, P. L. Atlas of tumor pathology: tumors of the salivary glands. $3^{a}$ série, fascículo 17 . Washington: Armed Forces Institute of Pathology, 1996.

10. EROL, B. et al. A massive pleomorphic adenoma of the submandibular salivary gland J Oral Maxillofac, v. 55, p. 1011-4, 1997

11. EVESON, J. W. et al. Pleomorfic adenoma. In: BARNES, L. et al. World Health Organization classification of tumours: pathology and genetics of head and neck tumours. Lyon: IARC Press, 2005.

12. EVESON, J. W.; CAWSON, R. A. Salivary gland tumours: a review of 2410 cases with particular reference to histological types, site, age and sex distribution. J Pathol, v. 146, p. 51-8, 1985.

13. FÉLIX, A. et al. Pleomorphic adenoma and carcinoma ex-pleomorphic adenoma: immunohistochemical demonstration of the association between tenascin expression and malignancy. Histopathol, v. 45, n. 6, p. 187-92, 2004.

14. FÉLIX, A. Laminin and collagen IV in pleomorphic adenoma and carcinoma ex-pleomorphic adenoma: an immunohistochemical study. Human Pathology, v. 30, n. 8, p. 964-9,1999.

15. FORTY, M. J.; WAKE, M. J. C. Pleomorphic salivary adenoma in an adolescent. British Dental J, v. 188, n. 10, p. 545-6, 2000.

16. FRIEDRICH, R.E. et al. Pleomorphic adenoma of the salivary glands: análisis of 94 patients. Anticancer Res, v. 25, n. 3A , p. 1703-05, 2005.

17. GARCIA-PERLA, A. et al. Pleomorphic adenoma of the parotid in childhood. J Craniomaxillofac Surg, v. 30, n. 4, p. 242-5, 2002.

18. HARADA, H. Histomorphological investigation regarding to malignant transformation of pleomorphic adenoma (so-called malignant mixed tumor) of the salivary gland origin: special reference carcinosarcoma. Kurume Med J, v. 47, n. 4, p. 307-23, 2000.

19. HILL, A. G. Major salivary gland tumours in a rural Kenyan hospital. East Afr Med J, v. 79, n. 1, p. 8-10, 2002.

20. ITO, F. A. et al. Salivary gland tumors in a brazilian population: a retrospective study of 496 cases. Int J Oral Maxillofac Surg, v. 34, n. 5, p. 533-6, 2005.

21. KAYEMBE, M. K.; KALENGAYI, M. M. Salivary gland tumours in Congo (Zaire). Odontostomatol Trop, v. 25, n. 99, p. 19-22, 2002.

22. LEWIS, J. E.; OLSEN, K. D; SEBO, J. Carcinoma ex pleomorphic adenoma: pathologic analysis of 73 cases. Human Pathology, v. 32, n. 6, p. 596-604, 2001. 
23. PARIS, J. et al. Pleomorphic adenoma of the parotid: hitopathological study. Ann Otolaryngol Chir Cervicofac, v. 121, n. 3, p. 161-6, 2004.

24. PINKSTON, J. A.; COLE, P. Incidence rates of salivary gland tumors: results from a pop based study. Otolaryngol Head Neck Surg, v. 120, n. 6, p. 834-40, 1999.

25. Ríos, P. C. et al. Parapharingeal parotid pleomorphic adenoma. Rev Port Orl, v. 38, n. 1, p. 57-9, 2000.

26. RYAN Jr., RE et al. Cellular mixed tumors of the salivary glands. Arch Otolaryngol, v. 104, n. 8, p. 451-3, 1978.

27. SANTOS, I. R. B.; KOWALSKI, L. P. Tumores benignos das glândulas parótidas: análise retrospectiva de uma série de 222 casos. Rev Bras Otorrinolaringol, v. 66, n. 2, p. 94-100, 2000.

28. SEIFERT, G. et al. Diseases of the salivary glands: diagnosis, pathology, treatment, facial nerve surgery. Stuttgart: Georg Thieme Verlag, 1986.

29. SILVA, S. J. et al. Estudo clínico-epidemiológico de 183 casos de neoplasias de glândulas salivares baseado na classificação da OMS (1991). Rev Bras Otorrinolaringol, v. 64, n. 4, p. 387-94, 1998.
30. SIMPSON, RHW. Classification of tumors of the salivary glands. Histopathol, v. 24, p. 187-91, 1994.

31. SPIRO, R. H. Salivary neoplasms: overview of 35-year experience with 2807 pacients. Head Neck Surg, v. 8, p. 177-84, 1986.

32. SUNARDHI-WIDYAPUTRA, S.; VAN DAME, B. Immunohistochemical expression of tenascin in normal human salivary glands and in pleomorphic adenomas. Pathol Res Pradt, v. 189, n. 2, p. 138-43, 1993.

33. SZMEJA, Z. et al. Pleomorphic adenomas in the materials of department of Otolaryngology of the Academy of Medical Sciences in Poznan. Otolaryngol Pol, v. 53, n. 5, p. 545-8, 1999.

34. VARGAS, P. A. et al. Salivary gland tumors in a Brazilian populatio: a retrospective study of 124 cases. Rev Hosp Clin Fac Med São Paulo, v. 57, n. 6, p. 271-6, 2002.

35. VARGHESE, B. T. et al. Pleomorphic adenoma of minor salivary gland in the parapharyngeal space. World $J$ Surg Oncol, v. 1, n. 1, p. 2, 2003. 